\title{
Extending Topological Abelian Groups by the Unit Circle
}

\author{
Hugo J. Bello, ${ }^{1}$ María Jesús Chasco, ${ }^{1}$ and Xabier Domínguez ${ }^{2}$ \\ ${ }^{1}$ Departamento de Física y Matemática Aplicada, University of Navarra, 31080 Pamplona, Spain \\ ${ }^{2}$ Departamento de Métodos Matemáticos y de Representación, University of A Coruña, 15071 A Coruña, Spain
}

Correspondence should be addressed to Xabier Domínguez; xabier.dominguez@udc.es

Received 26 June 2013; Accepted 30 August 2013

Academic Editor: Salvador Hernández

Copyright (C) 2013 Hugo J. Bello et al. This is an open access article distributed under the Creative Commons Attribution License, which permits unrestricted use, distribution, and reproduction in any medium, provided the original work is properly cited.

\begin{abstract}
A twisted sum in the category of topological Abelian groups is a short exact sequence $0 \rightarrow Y \rightarrow X \rightarrow Z \rightarrow 0$ where all maps are assumed to be continuous and open onto their images. The twisted sum splits if it is equivalent to $0 \rightarrow Y \rightarrow Y \times Z \rightarrow Z \rightarrow 0$. We study the class $S_{\mathrm{TG}}(\mathbb{T})$ of topological groups $G$ for which every twisted sum $0 \rightarrow \mathbb{T} \rightarrow X \rightarrow G \rightarrow 0$ splits. We prove that this class contains Hausdorff locally precompact groups, sequential direct limits of locally compact groups, and topological groups with $\mathscr{L}_{\infty}$ topologies. We also prove that it is closed by taking open and dense subgroups, quotients by dually embedded subgroups, and coproducts. As means to find further subclasses of $S_{\mathrm{TG}}(\mathbb{T})$, we use the connection between extensions of the form $0 \rightarrow \mathbb{T} \rightarrow X \rightarrow G \rightarrow 0$ and quasi-characters on $G$, as well as three-space problems for topological groups. The subject is inspired on some concepts known in the framework of topological vector spaces such as the notion of $\mathscr{K}$-space, which were interpreted for topological groups by Cabello.
\end{abstract}

\section{Introduction and Preliminaries}

In the theory of topological vector spaces (topological groups) a property $P$ is said to be a 3-space property if whenever a closed subspace (subgroup) $Y$ of a space (group) $X$ and the corresponding quotient $X / Y$ both have property $P, X$ also has property $P$.

A short exact sequence of topological vector spaces (topological groups) $0 \rightarrow Y \stackrel{\iota}{\rightarrow} X \stackrel{\pi}{\rightarrow} Z \rightarrow 0$ will be called a twisted sum, and the space (group) $X$ will be called an extension of $Z$ by $Y$ when both $\imath$ and $\pi$ are continuous and open onto their images. Using this language, 3-space properties can be described as those which are preserved by forming extensions.

An example of a 3-space property in the category of Banach spaces is reflexivity. However, the point-separating property (i.e., having a dual space which separates points) is not a 3 -space property. (Consider the space $l_{p}$ for $0<p<1$, and a weakly closed subspace $M$ of $l_{p}$ without the HahnBanach extension property. If we take as $N$ the kernel of some continuous linear functional on $M$ which does not extend to $l_{p}$, then $X=l_{p} / N$ does not have the point-separating property but both $L:=M / N$ and $X / L$ have this property (see $[1]))$.
In the category of topological Abelian groups, local compactness, precompactness, metrizability, and completeness are 3-space properties. However, $\sigma$-compactness, sequential completeness, realcompactness, and a number of other properties are not (see [2] for more examples).

The twisted sum $0 \rightarrow Y \stackrel{l}{\rightarrow} X \stackrel{\pi}{\rightarrow} Z \rightarrow 0$ splits if there exists a continuous linear map (continuous homomorphism) $T: X \rightarrow Y \times Z$ making the following diagram commutative $\left(\imath_{Y}\right.$ is the canonical inclusion of $Y$ into the product, and $\pi_{Z}$ is the canonical projection onto $Z$ ):

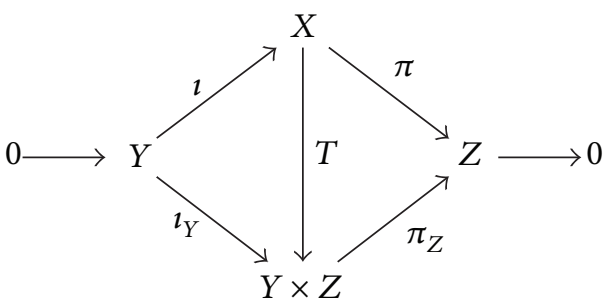

It is known that if such a $T$ exists, it must actually be a topological isomorphism. 
If the twisted sum $0 \rightarrow Y \stackrel{\iota}{\rightarrow} X \stackrel{\pi}{\rightarrow} Z \rightarrow 0$ splits and both $Y$ and $Z$ have a productive property $P$, then $X$ has property $P$, too.

Kalton et al. provided in [1] the first formal and extensive study of splitting twisted sums in the framework of $F$-spaces (complete metric linear spaces). They devote Chapter 5 of this monograph to the following problem: is local convexity a 3space property?

On the path to answering (in the negative) this question, the authors mention a useful result by Dierolf (1973) which asserts that there exists a non-locally-convex extension of the $F$-space $Z$ by the $F$-space $Y$ if and only if there exists a non-locally-convex extension of $Z$ by $\mathbb{R}$. The analogue of this result for topological groups, which involves the notion of local quasi-convexity, was obtained by Castillo in [3].

At this point the following definition, originally introduced in [4], comes across as natural: a $F$-space $X$ is said to be a $\mathscr{K}$-space if, whenever $Y$ is an $F$-space and $L$ is a subspace of $Y$ with dimension one such that $Y / L \cong X$, the corresponding twisted sum splits. The negative answer to the 3 -space problem for local convexity is obtained in [1] as a corollary of the fact that $\ell_{1}$ is not a $\mathscr{K}$-space.

The notion of $\mathscr{K}$-space is relevant on its own, regardless of 3-space properties. Many classical spaces such as $L_{p}(0<$ $p<\infty), l_{p}(p \neq 1)$, or $\mathcal{c}_{0}$ are $\mathscr{K}$-spaces.

In this paper we will study the natural counterpart of the notion of $\mathscr{K}$-space for topological groups and its connections with 3-space problems, following the work started by Cabello in [5-7].

For simplicity, and because our methods are applicable for the most part only to Abelian groups, we use additive notation, and denote by 0 the neutral element. We denote by $\mathbb{N}$ the set of all positive natural numbers, by $\mathbb{Z}$ the integers, by $\mathbb{R}$ the reals, by $\mathbb{C}$ the set of complex numbers, and by $\mathbb{T}$ the unit circle of $\mathbb{C}$, with the topology induced by $\mathbb{C}$. In $\mathbb{T}$ we will use multiplicative notation and we will denote by $p$ the canonical projection from $\mathbb{R}$ to $\mathbb{T}$ given by $p(t)=\exp (2 \pi i t)$. We will use $\mathcal{N}_{0}(G)$ to denote the system of neighborhoods of 0 in a topological Abelian group $G$.

Recall that a topological Abelian group $G$ is precompact if for every neighborhood of zero $V$ there exists a finite subset $F$ of $G$ such that $G=F+V$. Precompact groups are the subgroups of compact groups. In the same way a group $G$ is locally precompact if and only if it is a subgroup of a locally compact group.

The dual group $G^{\wedge}$ of a given topological Abelian group $G$ is formed from the continuous group homomorphisms from $G$ into $\mathbb{T}$, usually called characters. The polar set of a subset $A$ of $G$ is defined by $A^{0}:=\left\{\chi \in G^{\wedge}: \chi(A) \subseteq \mathbb{T}_{+}\right\}$, where $\mathbb{T}_{+}:=p([-1 / 4,1 / 4])=\{z \in \mathbb{T}: \operatorname{Re}(z) \geq 0\}$. A subset $A$ of a topological group $G$ is called quasi-convex if for every $x \in G \backslash$ $A$ there is a $\chi \in A^{0}$ such that $\chi(x) \notin \mathbb{T}_{+}$. A topological group is called locally quasi-convex if it has a neighborhood basis of 0 consisting of quasi-convex sets. It is well known (see [8]) that a topological vector space is locally convex if and only if it is a locally quasi-convex topological group in its additive structure.
If $G^{\wedge}$ separates the points of $G$ we say that $G$ is maximally almost periodic (MAP). Every locally quasi-convex group is a MAP group.

The analogous notion to the HBEP (Hahn-Banach Extension Property) for topological groups is the following. A subgroup $H \leq X$ is dually embedded in $X$ if each character of $H$ can be extended to a character of $X$.

When endowed with the compact-open topology $\tau_{\mathrm{co}}$, $G^{\wedge}$ becomes a Hausdorff topological group. A basis of neighborhoods of the neutral element for the compact open topology $\tau_{\text {co }}$ is given by the sets $K^{0}=\left\{\chi \in G^{\wedge}: \chi(K) \subseteq \mathbb{T}_{+}\right\}$, where $K$ is a compact subset of $G$.

Remark 1. Observe that a necessary condition for the splitting of the twisted sum of topological Abelian groups $0 \rightarrow H \stackrel{\imath}{\rightarrow}$ $X \stackrel{\pi}{\rightarrow} G \rightarrow 0$ is that $i(H)$ be a dually embedded subgroup of $X$.

The following known characterization is essential when dealing with twisted sums in different categories (see [3, Lemma 3.1] for a proof).

Theorem 2. Let $0 \rightarrow H \stackrel{\imath}{\rightarrow} X \stackrel{\pi}{\rightarrow} G \rightarrow 0$ be a twisted sum of topological Abelian groups. The following are equivalent:

(1) $0 \rightarrow H \stackrel{\imath}{\rightarrow} X \stackrel{\pi}{\rightarrow} G \rightarrow 0$ splits;

(2) there exists a continuous homomorphism $S: G \rightarrow X$ with $\pi \circ S=\mathrm{id}_{G}$, i.e., a right inverse for $\pi$;

(3) there exists a continuous homomorphism $P: X \rightarrow H$ with $P \circ \imath=\mathrm{id}_{H}$, i.e., a left inverse for $\imath$.

We will use the notions of pull-back and push-out in the category of topological Abelian groups, following Castillo [3]. Given topological Abelian groups $A, B$, and $C$ and continuous homomorphisms $u: A \rightarrow B$ and $v: A \rightarrow C$, the push-out of $u$ and $v$ is a topological group PO and two continuous homomorphisms $r$ and $s$ making the square diagram commutative

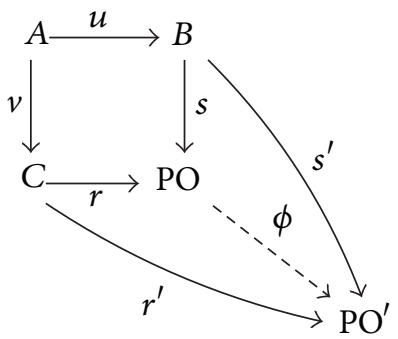

and such that for every topological Abelian group $\mathrm{PO}^{\prime}$ and continuous homomorphisms $r^{\prime}: C \rightarrow \mathrm{PO}^{\prime}$ and $s^{\prime}: B \rightarrow$ $\mathrm{PO}^{\prime}$ with $s^{\prime} \circ u=r^{\prime} \circ v$, there is a unique continuous homomorphism $\phi$ from $\mathrm{PO}$ to $\mathrm{PO}^{\prime}$ making the two triangles commutative. The topological group PO exits and is unique up to topological isomorphism.

Given any twisted sum $0 \rightarrow H \stackrel{\imath}{\rightarrow} X \stackrel{\pi}{\rightarrow} G \rightarrow 0$ of topological Abelian groups, any topological Abelian group $Y$, 
and any continuous homomorphism $t: H \rightarrow Y$, if $\mathrm{PO}$ is the push-out of $t$ and $t$, there is a commutative diagram

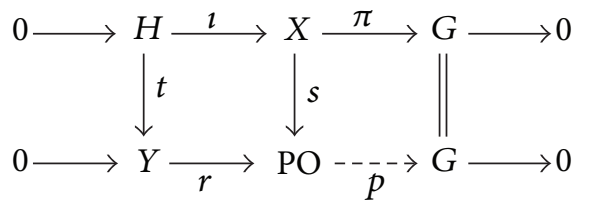

where both squares are commutative and the bottom sequence is a twisted sum [3].

An analogous result for the dual construction (the pullback) can be obtained (see [3]).

Lemma 3. Let $K$ be a compact subgroup of a topological Abelian group $X . X^{\wedge}$ separates points of $K$ if and only if $K$ is dually embedded in $X$.

Proof. Suppose that $X^{\wedge}$ separates points of $K$. It is known that for any locally compact Abelian group $G$, a subgroup $L \leq G^{\wedge}$ is dense in $G^{\wedge}$ if and only if it separates points of $G$ (see [9, Proposition 31]). The subgroup $L$ of $K^{\wedge}$ formed by all restrictions of characters of $X$ separates points of $K$ by hypothesis. Hence $L$ is dense in $K^{\wedge}$ and, as $K^{\wedge}$ is discrete, $L$ coincides with $K^{\wedge}$.

Suppose that $K$ is dually embedded in $X$. As $K$ is compact, it is a MAP group. Fix a nonzero $x \in K$. There exists $\chi \in K^{\wedge}$ such that $\chi(x) \neq 1$. Since $K$ is dually embedded in $X$, there exists an extension $\tilde{\chi} \in G^{\wedge}$ of $\chi$ with $\tilde{\chi}(x)=\chi(x) \neq 1$.

Corollary 4 ([10, Proposition 1.4]). Let $K$ be a compact subgroup of a topological Abelian group $X$. If $X$ is maximally almost periodic, then $K$ is dually embedded in $X$.

\section{The Class $S_{\mathrm{TG}}(\mathbb{T})$}

Next we consider the particular case in which the compact subgroup $K$ is $\mathbb{T}$. (The proofs of Theorems 5 and 7 below can be extracted from that of [3, Theorem 4.1], but we prefer the present formulation.)

Theorem 5. Let $0 \rightarrow \mathbb{1} \stackrel{\imath}{\rightarrow} X \stackrel{\pi}{\rightarrow} G \rightarrow 0$ be a twisted sum of topological Abelian groups. The following are equivalent:

(1) the twisted sum $0 \rightarrow \mathbb{T} \stackrel{\imath}{\rightarrow} X \stackrel{\pi}{\rightarrow} G \rightarrow 0$ splits;

(2) $X^{\wedge}$ separates points of $\imath(\mathbb{T})$;

(3) $\imath(\mathbb{T})$ is dually embedded in $X$.

Proof. $2 \Leftrightarrow 3$ is a corollary of Lemma 3 .

$3 \Rightarrow 1$ : suppose that $\imath(\mathbb{T})$ is dually embedded in $X$. Hence there exists a continuous character $\chi: X \rightarrow \mathbb{W}$ which extends the isomorphism $\varphi: \imath(\mathbb{T}) \rightarrow \mathbb{T}$ defined by $\varphi(\imath(t))=t$. Since $\chi \circ \imath=\mathrm{id}_{\mathbb{T}}$, the assertion follows from Theorem 2 .

$1 \Rightarrow 2$ : fix $x \in \imath(\mathbb{T}), x=\imath(z)$ with $z \neq 1$. By Theorem 2, there exists a continuous homomorphism $P: X \rightarrow \mathbb{T}$ with $P \circ \imath=\mathrm{id}_{\mathbb{T}}$; hence $P(\imath(z))=\mathrm{id}_{\mathbb{T}}(z)=z \neq 1$.

Now we will complete the previous Theorem under the assumption that $G$ is locally quasi-convex. We will use the following result due to Castillo, concerning the 3-space problem in locally quasi-convex groups.

Lemma 6 ([3, Theorem 2.1]). Let $H$ be a locally quasi-convex subgroup of a topological Abelian group $X$ such that $X / H$ is locally quasi-convex. Then $X$ is locally quasi-convex if and only if $H$ is dually embedded in $X$.

Theorem 7. Let $0 \rightarrow \mathbb{T} \stackrel{\imath}{\rightarrow} X \stackrel{\pi}{\rightarrow} G \rightarrow 0$ be a twisted sum of topological Abelian groups. Suppose that $G$ is locally quasiconvex. Then conditions (1), (2), (3) of Theorem 5 are equivalent to

(4) $X$ is locally quasi-convex.

Proof. $1 \Rightarrow 4$ : if the twisted sum splits, $X$ is topologically isomorphic to the product of two locally quasi-convex groups; hence it is locally quasi-convex.

$4 \Rightarrow 3$ : given a twisted sum $0 \rightarrow \mathbb{\mathbb { V }} \stackrel{\imath}{\rightarrow} X \stackrel{\pi}{\rightarrow} G \rightarrow 0$ with $X$ and $G$ locally quasi-convex, since $G \cong X / \imath(\mathbb{T})$, by Lemma 6 , we deduce that $\imath(\mathbb{T})$ is dually embedded in $X$.

Following the notation used by Domański [11] in the framework of topological vector spaces, we introduce the class $S_{\mathrm{TG}}(\mathbb{T})$ which is the analogue of that of $\mathscr{K}$-spaces for topological Abelian groups.

Definition 8. We say that a topological Abelian group $G$ is in the class $S_{\mathrm{TG}}(\mathbb{T})$ if every twisted sum of topological Abelian groups $0 \rightarrow \mathbb{T} \rightarrow X \rightarrow G \rightarrow 0$ splits.

Theorem 9. Let $G$ be a topological vector space such that $G \in$ $S_{\mathrm{TG}}(\mathbb{T})$ as a topological group. Then $G$ is a $\mathscr{K}$-space.

Proof. Suppose that $G \in S_{\mathrm{TG}}(\mathbb{T})$. Fix a twisted sum of topological vector spaces $0 \rightarrow \mathbb{R} \stackrel{\imath}{\rightarrow} X \stackrel{\pi}{\rightarrow} G \rightarrow 0$. Recall that we denote by $p: \mathbb{R} \rightarrow \mathbb{T}$ the canonical projection, given by $p(r)=\exp (2 \pi i r)$. If we consider the push-out PO of $p$ and $\imath$, we obtain a commutative diagram

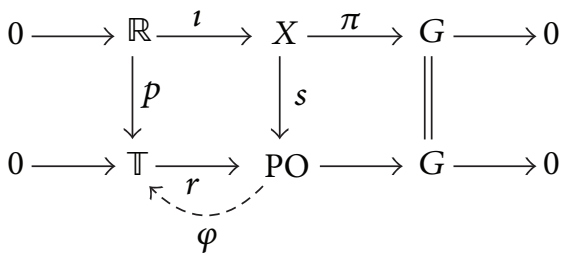

where the bottom sequence is a twisted sum. Since $G \in$ $S_{\mathrm{TG}}(\mathbb{T})$, this sequence splits. Hence there exists a left inverse $\varphi$ for $r$. Then $\varphi \circ s \circ \imath=p$. Since $X$ is a topological vector space, $\varphi \circ s$ is of the form $x \mapsto \exp (2 \pi i f(x))$ for some continuous linear functional $f \in X^{*}$. This clearly implies $f \circ \imath=\mathrm{id}_{\mathbb{R}}$; that is, $f$ is a left inverse for $\imath$; hence the top sequence splits, too.

Theorem $10([4,12,13])$. There is a short exact sequence of topological vector spaces and continuous, relatively open linear maps $0 \rightarrow \mathbb{R} \stackrel{\iota}{\rightarrow} X \stackrel{\pi}{\rightarrow} G \rightarrow 0$ which does not split. In other words, $\ell_{1}$ is not a $\mathscr{K}$-space.

Using Theorem 9 we deduce the following. 
Corollary 11. $\ell_{1} \notin S_{\mathrm{TG}}(\mathbb{T})$.

Remark 12. The above corollary gives an example of a quotient $X / \mathbb{T}$ which is locally quasi-convex as a topological group but such that $X$ does not even separate points of $\mathbb{T}$ : a strong failure of the 3-space property for local quasi-convexity and for the property of being a MAP group.

From Theorem 5 it follows that a topological Abelian group $G$ is in $S_{\mathrm{TG}}(\mathbb{T})$ iff for every twisted sum of topological Abelian groups of the form $0 \rightarrow \mathbb{T} \stackrel{\imath}{\rightarrow} X \stackrel{\pi}{\rightarrow} G \rightarrow 0$, the subgroup $\imath(\mathbb{T})$ is dually embedded in $X$. Note that in any such twisted sum, for an arbitrary $A \leq G$ the subgroup $\pi^{-1}(A)$ contains $\imath(\mathbb{T})$. This yields the following criterion;

Proposition 13. Let $G$ be a topological Abelian group. Suppose that there exists a subgroup $A \leq G$ which is in $S_{\mathrm{TG}}(\mathbb{T})$ and satisfies the following property. For every twisted sum of topological Abelian groups of the form $0 \rightarrow \mathbb{T} \stackrel{\imath}{\rightarrow} X \stackrel{\pi}{\rightarrow}$ $G \rightarrow 0$, the subgroup $\pi^{-1}(A)$ is dually embedded in $X$. Then $G \in S_{\mathrm{TG}}(\mathbb{T})$.

Corollary 14. Let $G$ be a topological Abelian group. Suppose that there exists an open subgroup $A \leq G$ such that $A \in$ $S_{\mathrm{TG}}(\mathbb{T})$. Then $G \in S_{\mathrm{TG}}(\mathbb{T})$.

Proof. If $A$ is an open subgroup of $G$, with the notation of Proposition $13, \pi^{-1}(A)$ is an open subgroup of $X$ and hence dually embedded.

Corollary 15. Let $G$ be a topological Abelian group. Suppose that there exists a dense subgroup $A \leq G$ such that $A \in S_{\mathrm{TG}}(\mathbb{T})$. Then $G \in S_{\mathrm{TG}}(\mathbb{\mathbb { T }})$.

Proof. If $A$ is a dense subgroup of $G$, with the notation of Proposition $13, \pi^{-1}(A)$ is a dense subgroup of $X$ because $\pi$ is continuous and onto. In particular it is dually embedded in $X$.

We next see that the converse of Corollary 15 is true in the case of $G$ metrizable. For any topological Abelian group $G$, we denote by $\widetilde{G}$ the Raikov completion of $G$. See [14] for more information about this subject.

Lemma 16 ([14, Theorem 6.11]). If $G$ is a metrizable topological group that has a completion $\widetilde{G}$ and if $H$ is a closed normal subgroup of $G$, then $G / H$ has a completion that is topologically isomorphic to $\widetilde{G} / \widetilde{H}$, where $\widetilde{H}$ is the closure of $H$ in $\widetilde{G}$.

Proposition 17. Let $G$ be a metrizable topological Abelian group which is in $S_{\mathrm{TG}}(\mathbb{T})$. Suppose that $A$ is a dense subgroup of $G$. Then $A$ is in $S_{\mathrm{TG}}(\mathbb{T})$, too.

Proof. Let $X$ be a topological Abelian group, and let $\mathbb{T} \cong L \leq$ $X$ be a subgroup such that $A \cong X / L$. Since metrizability is a 3 -space property $([15,5.38(\mathrm{e})]), X$ is a metrizable group. By Lemma $16, \widetilde{G}=\widetilde{A} \cong \widetilde{X} / L$. By Corollary $15, \widetilde{G} \in S_{\mathrm{TG}}(\mathbb{T})$. It follows that $L$ is dually embedded in $\widetilde{X}$; hence it is dually embedded in $X$, too.
Our next aim is to prove that Hausdoff locally precompact groups are in $S_{\mathrm{TG}}(\mathbb{T})$. Note first that if $G$ is a topological Abelian group and $H \leq G$ is a precompact subgroup such that the quotient $G / H$ is locally precompact, then $G$ is locally precompact, too. (Indeed, let $\pi: G \rightarrow G / H$ be the canonical projection. Choose $U \in \mathcal{N}_{0}(G)$ such that $\pi(U)$ is precompact. Let us see that $U$ is precompact. Given $V \in \mathcal{N}_{0}(G)$ we need to find a finite subset $F \subset G$ with $U \subset F+V$. Fix $V^{\prime} \in \mathcal{N}_{0}(G)$ with $V^{\prime}+V^{\prime} \subset V$ and find a finite $F_{1} \subset G$ with $H \subset F_{1}+V^{\prime}$. Since $\pi(U)$ is precompact there exists a finite subset $F_{2}^{\prime}$ of $G / H$ with $\pi(U) \subset F_{2}^{\prime}+\pi\left(V^{\prime}\right)$. We may suppose that $F_{2}^{\prime}=\pi\left(F_{2}\right)$ with $F_{2}$ being a finite subset of $G$. Hence $\pi(U) \subset \pi\left(F_{2}\right)+\pi\left(V^{\prime}\right)=$ $\pi\left(F_{2}+V^{\prime}\right)$, which implies $U \subset \pi^{-1} \pi(U) \subset \pi^{-1} \pi\left(F_{2}+V^{\prime}\right)=F_{2}+$ $V^{\prime}+H \subset F_{1}+F_{2}+V^{\prime}+V^{\prime} \subset F+V$ if we put $F=F_{1}+F_{2}$.)

Theorem 18. Locally precompact Hausdorff Abelian groups are in $S_{\mathrm{TG}}(\mathbb{T})$.

Proof. Let $G$ be a locally precompact Hausdorff Abelian group. Given a twisted sum $0 \rightarrow \mathbb{T} \stackrel{\iota}{\rightarrow} X \stackrel{\pi}{\rightarrow} G \rightarrow 0$, as $\mathbb{T}$ is in particular precompact, by the above argument $X$ is locally precompact, too. But every subgroup of a locally compact group is dually embedded $[15,24.12]$, so $\imath(\mathbb{T})$ is dually embedded in $\widetilde{X}$, the completion of $X$. Then $\imath(\mathbb{T})$ is also dually embedded in $X$ and by Theorem 5 , the twisted sum splits.

Corollary 19. Locally compact Hausdorff Abelian groups and precompact Hausdorff Abelian groups are in $S_{\mathrm{TG}}(\mathbb{T})$.

Remark 20. It is proved in [11] that every topological vector space endowed with its weak topology is a $\mathscr{K}$-space. The above corollary shows that a similar result is true for topological Abelian groups, since a topological group endowed with the topology induced by its characters is precompact (see [16]).

Theorem 21. Let $G$ be a topological Abelian group.

(1) If a closed subgroup $H \leq G$ is such that $G / H \in S_{\mathrm{TG}}$ (T), then $H$ is dually embedded.

(2) If $\mathrm{G}$ is in $S_{\mathrm{TG}}(\mathbb{T})$ and $H \leq G$ is a closed dually embedded subgroup, then $G / H \in S_{\mathrm{TG}}(\mathbb{T})$.

Proof. (1) Suppose that $G / H \in S_{\mathrm{TG}}(\mathbb{T})$. Let $\chi: H \rightarrow \mathbb{T}$ be a character, and consider the natural twisted sum $0 \rightarrow H \stackrel{\bullet}{\rightarrow}$ $G \stackrel{\pi}{\rightarrow} G / H \rightarrow 0$. By taking the corresponding push out, we obtain the following commutative diagram:

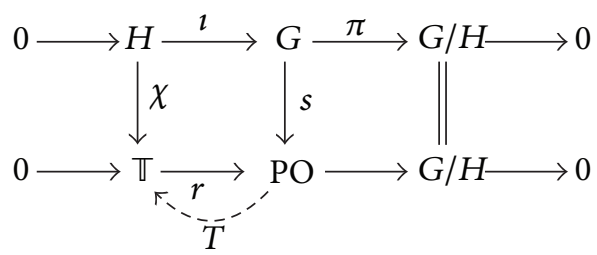

where both rows are twisted sums of topological Abelian groups. Since $G / H \in S_{\mathrm{TG}}(\mathbb{T})$, the bottom sequence splits; 
hence by Theorem 2, there exists a continuous homomorphism $T: P O \rightarrow \mathbb{T}$ such that $T \circ r=\mathrm{id}_{\mathbb{T}}$. The homomorphism $T \circ s$ is an extension of $\chi$.

(2) Suppose that $H$ is dually embedded in $G$. Fix a twisted sum $0 \rightarrow \mathbb{T} \stackrel{\imath}{\rightarrow} X \stackrel{\pi}{\rightarrow} G / H \rightarrow 0$. Let $q: G \rightarrow G / H$ be the canonical projection, and let $\mathrm{PB}=\{(g, x) \in G \times X$ : $q(g)=\pi(x)\}$ be the pull-back of $q$ and $\pi$. Define $r: \mathrm{PB} \rightarrow G$ and $s: \mathrm{PB} \rightarrow X$, as the restrictions of the corresponding projections. Note that

$$
\begin{aligned}
\text { ker } r & =\{(g, x) \in \mathrm{PB}: r(g, x)=0\}=\{(g, x) \in \mathrm{PB}: g=0\} \\
& =\{(0, x) \in G \times X: H=\pi(x)\} \\
& =\{0\} \times \imath(\mathbb{T}), \\
\text { ker } s & =\{(g, x) \in \mathrm{PB}: s(g, x)=0\}=\{(g, x) \in \mathrm{PB}: x=0\} \\
& =\{(g, 0) \in G \times X: q(g)=H\} \\
& =H \times\{0\} .
\end{aligned}
$$

We thus obtain the following diagram:

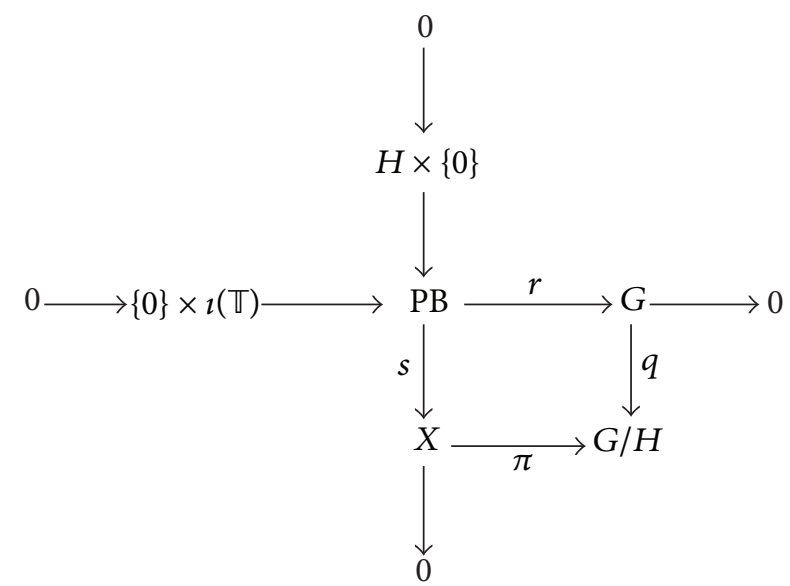

Note that since $q$ and $\pi$ are onto, the definition of $\mathrm{PB}$ yields $r((U \times X) \cap \mathrm{PB}) \supset U$ and $s((G \times V) \cap \mathrm{PB}) \supset V$ for every $U \in \mathcal{N}_{0}(G)$ and $V \in \mathcal{N}_{0}(X)$; hence $r$ and $s$ are onto and open. Thus both short sequences are twisted sums of topological Abelian groups.

Fix $\varphi \in \imath(\mathbb{T})^{\wedge}$; we will find an extension of $\varphi$ to the whole $X$. We can regard $\varphi$ as a character of $\{0\} \times \imath(\mathbb{T})$ by defining $\widetilde{\varphi}(0, x)=\varphi(x)$. Since $\{0\} \times \imath(\mathbb{T}) \cong \mathbb{T}$ and $G \in S_{\mathrm{TG}}(\mathbb{T})$, by Theorem $5,\{0\} \times \imath(\mathbb{T})$ is dually embedded in PB. Thus there exists $\psi \in \mathrm{PB}^{\wedge}$ with $\left.\psi\right|_{\{0\} \times i(\mathbb{T})}=\widetilde{\varphi}$; that is, $\psi(0, x)=\varphi(x)$ for every $x \in \imath(\mathbb{T})$. Define, for every $h \in H, \widetilde{\psi}(h)=\psi(h, 0)$ (note that if $h \in H$ then $(h, 0) \in \mathrm{PB})$.

Since $H$ is dually embedded in $G$ there exists $\sigma \in G^{\wedge}$ with $\sigma_{\mid H}=\widetilde{\psi}$ i.e. $\sigma(h)=\psi(h, 0)$ for every $h \in H$. Now define $\rho \in \mathrm{PB}^{\wedge}$ as follows: $\rho(g, x)=\psi(g, x) \overline{\sigma(g)}$. This is clearly continuous. Note that ker $\rho \geq \operatorname{ker} s=H \times\{0\}$ since if $h \in H$, we have that $\rho(h, 0)=\psi(h, 0) \overline{\sigma(h)}=1$. As $X \cong \mathrm{PB} /(H \times\{0\})$, the character $\widetilde{\rho}$ in $X^{\wedge}$ given by $\widetilde{\rho}(x)=\rho(g, x)$ for every $(g, x) \in \mathrm{PB}$ is well defined and continuous.

Now $\tilde{\rho}$ is the desired extension of $\varphi$ : if $x \in \imath(\mathbb{T})$, we have $\tilde{\rho}(x)=\rho(0, x)=\psi(0, x) \overline{\sigma(0)}=\psi(0, x)=\varphi(x)$.

Remark 22. An analogous result in the framework of $F$ spaces is [1, Theorem 5.2] (cf. also [11, Lemma 4.1]).

The following corollary is a generalization of Theorems 5.2 and 5.3 in [4] and appears as Theorem 4.1 in [3].

Corollary 23. A topological Abelian group $G$ is in $S_{\mathrm{TG}}(\mathbb{T})$ if and only if whenever $X$ is a topological Abelian group and $H$ is a closed subgroup of $X$ with $X / H \cong G$, then $H$ is dually embedded.

Proof. This follows from Theorems 21(1) and 5.

Let $\left(G_{\alpha}\right)_{\alpha \in I}$ be a family of topological Abelian groups. The coproduct of $\left(G_{\alpha}\right)_{\alpha \in I}$ is the direct sum $\bigoplus_{\alpha \in I} G_{\alpha}$ endowed with the finest group topology making the inclusion maps $i_{\beta}: G_{\beta} \hookrightarrow \bigoplus_{\alpha \in I} G_{\alpha}$ continuous, for every $\beta \in I$. If $\left(G_{n}\right)_{n=1}^{\infty}$ is a countable family of groups, this topology coincides with the box topology on $\bigoplus_{n \in \mathbb{N}} G_{n}$.

Recall that the coproduct $\bigoplus_{\alpha \in I} G_{\alpha}$ has the following universal property. Given an arbitrary topological Abelian group $G$ and a homomorphism $f: \bigoplus_{\alpha \in I} G_{\alpha} \rightarrow G, f$ is continuous if and only if $f \circ i_{\beta}$ is continuous $\forall \beta \in I$.

Proposition 24. Let $\left(G_{\alpha}\right)_{\alpha \in I}$ be a family of topological Abelian groups in $S_{\mathrm{TG}}(\mathbb{T})$. The coproduct $\bigoplus_{\alpha \in I} G_{\alpha}$ is in $S_{\mathrm{TG}}(\mathbb{T})$.

Proof. Let $0 \rightarrow \mathbb{T} \stackrel{\imath}{\rightarrow} X \stackrel{\pi}{\rightarrow} \bigoplus_{\alpha \in I} G_{\alpha} \rightarrow 0$ be a twisted sum. Consider, for each $\beta \in I$, the pull-back $\mathrm{PB}_{\beta}$ of $\pi$ and $i_{\beta}: G_{\beta} \rightarrow \bigoplus_{\alpha \in I} G_{\alpha}$; for every $\beta \in I$ there is a commutative diagram

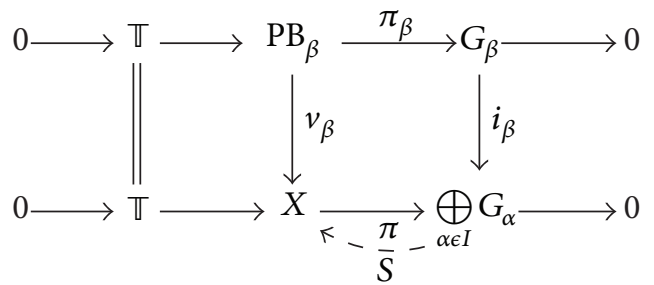

whose rows are twisted sums. As $G_{\beta} \in S_{\mathrm{TG}}(\mathbb{T})$, by Theorem 2, there exists a homomorphism $S_{\beta}: G_{\beta} \rightarrow \mathrm{PB}_{\beta}$ such that $\pi_{\beta} \circ S_{\beta}=\mathrm{id}_{G_{\beta}}$. Consider the map

$$
\begin{aligned}
S: & \bigoplus_{\alpha \in I} G_{\alpha} \longrightarrow X \\
& \left(g_{\alpha}\right)_{\alpha \in I} \longmapsto \sum_{\alpha \in I} v_{\alpha} \circ S_{\alpha}\left(g_{\alpha}\right) .
\end{aligned}
$$


As $v_{\alpha} \circ S_{\alpha}$ is a continuous homomorphism, by the universal property of the coproduct, $S$ is a continuous homomorphism. For every $g=\left(g_{\alpha}\right)_{\alpha \in I} \in \bigoplus_{\alpha \in I} G_{\alpha}$, we have

$$
\begin{aligned}
\pi(S(g)) & =\pi\left(\sum_{\alpha \in I} v_{\alpha} \circ S_{\alpha}\left(g_{\alpha}\right)\right) \\
& =\sum_{\alpha \in I}\left(\pi \circ v_{\alpha} \circ S_{\alpha}\right)\left(g_{\alpha}\right)=\sum_{\alpha \in I} i_{\alpha}\left(g_{\alpha}\right)=g,
\end{aligned}
$$

so $S$ is a right inverse for $\pi$, and again by Theorem 2 , the initial twisted sum splits.

The class of nuclear groups was formally introduced by Banaszczyk in [8]. His aim was to find a class of topological groups enclosing both nuclear spaces and locally compact Abelian groups (as natural generalizations of finitedimensional vector spaces). The original definition is rather technical, as could be expected from its success in gathering objects from such different classes into the same framework. Next we collect some facts concerning the class of nuclear groups which are relevant to this paper.

(i) Nuclear groups are locally quasi-convex $[8,8.5]$.

(ii) Subgroups of nuclear groups are dually embedded [8, 8.3].

(iii) Products, countable coproducts, subgroups, and Hausdorff quotients of nuclear groups are nuclear [8, $7.6,7.8,7.5]$.

(iv) Every locally compact Abelian group is nuclear [8, 7.10].

(v) A nuclear locally convex space is a nuclear group [8, 7.4]. Furthermore, if a topological vector space is a nuclear group, then it is a locally convex nuclear space $[8,8.9]$.

Theorem 25. Let $\left\{G_{n}, f_{n}^{m}\right\} \quad(n \leq m)$ be a countable direct system of nuclear Abelian groups in $S_{\mathrm{TG}}(\mathbb{T})$. Then the direct limit $\lim _{\rightarrow} G_{n}$ is in $S_{\mathrm{TG}}(\mathbb{T})$. In particular, sequential direct limits of locally compact groups are in $S_{\mathrm{TG}}(\mathbb{T})$.

Proof. The direct sum $\bigoplus_{n=1}^{\infty} G_{n}$ with the coproduct topology is in $S_{\mathrm{TG}}(\mathbb{T})$ by Proposition 24. Let $i_{m}: G_{m} \rightarrow \bigoplus G_{n}$ be the inclusion map, for every $m \in \mathbb{N}$. It is known (see [17]) that $\lim _{\rightarrow} G_{n} \cong\left(\bigoplus G_{n}\right) / \bar{H}$, where $\bar{H}$ is the closure of the subgroup $H$ generated by $\left\{i_{m} \circ f_{n}^{m}\left(g_{n}\right)-i_{n}\left(g_{n}\right): n \leq m ; g_{n} \in G_{n}\right\}$. Since countable coproducts of nuclear groups are nuclear groups, $\bar{H}$ is dually embedded. By Theorem $21, \lim _{\rightarrow} G_{n} \in S_{\mathrm{TG}}(\mathbb{T})$.

Varopoulos introduced in [18] the class $\mathscr{L}_{\infty}$ of all topological groups whose topologies are the intersection of a decreasing sequence of locally compact Hausdorff group topologies. He succeeded in his aim of extending known results about locally compact groups and established the basis for the development of the harmonic analysis on $\mathscr{L}_{\infty}$ groups. Subsequently many other authors investigated different properties of this class ([19-23]).

The following is a relevant fact concerning the structure of $\mathscr{L}_{\infty}$ groups proved by Sulley.
Proposition 26 ([22]). Let $G$ be any Abelian group endowed with an $\mathscr{L}_{\infty}$ topology. Then $G$ has an open subgroup which is a strict inductive limit of a sequence of Hausdorfflocally compact Abelian groups.

Corollary 27. Let $G$ be any Abelian group endowed with an $\mathscr{L}_{\infty}$ topology. Then $G$ is in $S_{\mathrm{TG}}(\mathbb{T})$.

Proof. By the above proposition and Theorem 25, $G$ has an open subgroup in $S_{\mathrm{TG}}(\mathbb{T})$. Hence Corollary 14 implies that $G$ is in $S_{\mathrm{TG}}(\mathbb{T})$.

\section{Quasi-Homomorphisms}

In his study of the stability of homomorphisms between topological Abelian groups [7], Cabello defined the notion of quasi-homomorphism, which is inspired by the technique of quasi-linear maps introduced by Kalton and others (see [1]).

Definition 28 ([7]). Let $G$ and $H$ be topological Abelian groups and $\omega: G \rightarrow H$ a map with $\omega(0)=0$. We say that $\omega$ is a quasi-homomorphism if the map $\Delta_{\omega}:(x, y) \in G \times G \mapsto$ $\omega(x+y)-\omega(x)-\omega(y) \in H$ is continuous at $(0,0)$.

A quasi-homomorphism $\omega: G \rightarrow H$ is approximable if there exists a homomorphism $a: G \rightarrow H$ such that $\omega-a$ is continuous at 0 .

Our aim is to use the notion of approximable quasihomomorphisms to obtain new examples of groups in $S_{\mathrm{TG}}(\mathbb{T})$.

We start with some facts about quasi-homomorphism taken from [7].

Proposition 29. Let $G$ and $H$ be topological Abelian groups and $\omega: G \rightarrow H$ a quasi-homomorphism.

(1) The sets

$$
\begin{array}{r}
W(V, U)=\{(h, g) \in H \times G: g \in U, h \in \omega(g)+V\} \\
\left(U \in \mathcal{N}_{0}(G), V \in \mathcal{N}_{0}(H)\right)
\end{array}
$$

form a basis of neighborhoods of zero for a group topology on $H \times G$.

(2) If $H \bigoplus_{\omega} G$ denotes the group $H \times G$ endowed with the topology induced by the quasi-homomorphism $\omega$ and $\imath_{H}$ and $\pi_{G}$ denote the canonical inclusion and projection, respectively, $0 \rightarrow H \stackrel{{ }^{l_{H}}}{\longrightarrow} H \bigoplus_{\omega} G \stackrel{\pi_{G}}{\longrightarrow} G \rightarrow$ 0 is a twisted sum of topological Abelian groups.

(3) A quasi-homomorphism is approximable if and only if the induced twisted sum splits.

(4) The twisted sum $0 \rightarrow H \stackrel{\imath}{\rightarrow} X \stackrel{\pi}{\rightarrow} G \rightarrow 0$ is equivalent to one induced by a quasi-homomorphism if and only if it splits algebraically and there exists a map $\rho: G \rightarrow X$ such that $\pi \circ \rho=\mathrm{id}_{G}, \rho(0)=0$ and $\rho$ is continuous at the origin.

Lemma 30. Let $G$ and $H$ be topological Abelian groups and $\omega: G \rightarrow H$ a quasi-homomorphism. Then the map $g \in G \mapsto$ $(\omega(g), g) \in H \bigoplus_{\omega} G$ is continuous at 0 . 
Proof. Note that for every $U \in \mathcal{N}_{0}(G)$ and $V \in \mathcal{N}_{0}(H)$ we have $[g \in U \Rightarrow(\omega(g), g) \in W(V, U)]$.

The following result is [7, Lemma 11]. We give here a proof for the sake of completeness.

Proposition 31. Let $\pi: X \rightarrow G$ be a continuous and open surjective homomorphism between topological Abelian groups. Suppose that $X$ is metrizable. Then there exists $\rho: G \rightarrow X$ such that $\pi \circ \rho=\mathrm{id}_{G}$ and $\rho$ is continuous at $0 \in G$.

Proof. Note that in order to define $\rho$ with $\pi \circ \rho=\mathrm{id}_{G}$, we simply must choose for every $g \in G$ an element $x \in \pi^{-1}(g)$, which is a nonempty set since $\pi$ is onto. Let us see that it can be done in such a way that the map thus obtained is continuous at zero.

Let $\left\{U_{n}: n \in \mathbb{N}\right\}$ be a decreasing basic sequence of neighborhoods of zero in $X$, where $U_{1}=X$. Due to the continuity of $\pi$, we have $\bigcap_{n \in \mathbb{N}} \pi\left(U_{n}\right)=\{0\}$. Let $\rho$ take the value 0 on $g=0$. For any $g \neq 0$, by the previous paragraph, we can choose $n$ and $x$ with $\pi(x)=g, x \in U_{n}, g \notin \pi\left(U_{n+1}\right)$, and define $\rho(g)=x$. Now fix $m \in \mathbb{N}$; we must find $V \in \mathcal{N}_{0}(G)$ with $\rho(V) \subseteq U_{m}$. Since $\pi$ is open there exists $V \in \mathcal{N}_{0}(G)$ with $\pi\left(U_{m}\right) \supseteq V$. Fix $g \in V$, and let us show that $\rho(g) \in U_{m}$. If $\rho(g)=0$ this is trivial. Otherwise $\rho(g)=x$ with $\pi(x)=$ $g, x \in U_{n}, g \notin \pi\left(U_{n+1}\right)$ for some $n$. Then $g \in V \subseteq \pi\left(U_{m}\right)$; hence $m \leq n$ and $x \in U_{n} \subseteq U_{m}$.

Corollary 32. Let $G$ be a metrizable topological Abelian group.

(1) If $H$ is metrizable and divisible, every twisted sum $0 \rightarrow$ $H \stackrel{\imath}{\rightarrow} X \stackrel{\pi}{\rightarrow} G \rightarrow 0$ is equivalent to one induced by $a$ quasi-homomorphism.

(2) $G$ is in $S_{\mathrm{TG}}(\mathbb{T})$ if and only if every quasi-character $\omega$ : $G \rightarrow \mathbb{T}$ is approximable.

Proof. Metrizability is a 3-space property (see $[15,5.38(\mathrm{e})]$ ). If $0 \rightarrow H \stackrel{\imath}{\rightarrow} X \stackrel{\pi}{\rightarrow} G \rightarrow 0$ is a twisted sum where both $G$ and $H$ are metrizable, so is $X$, and thus the hypotheses of Proposition 31 hold. Therefore, there exists a section of $\pi$ continuous at the origin. As $H$ is a divisible group, the twisted sum $0 \rightarrow H \stackrel{\imath}{\rightarrow} X \stackrel{\pi}{\rightarrow} G \rightarrow 0$ splits algebraically. By Proposition 29(4), this twisted sum is equivalent to one induced by a quasi-homomorphism.

The second part is a consequence of the first one and Proposition 29(3).

We call a norm on an Abelian group $G$ any subadditive, symmetric functional $\|\cdot\|: G \rightarrow[0, \infty)$ such that $\|x\|=0$ if and only if $x=0$.

Definition 33. For any $t \in \mathbb{T}$ let us call $\langle t\rangle$ the only real number $\alpha \in]-1 / 2,1 / 2]$ such that $\exp (2 \pi i \alpha)=t$. Then $[t \rightarrow|\langle t\rangle|]$ is a norm on $\mathbb{T}$. (Note that $\langle t s\rangle \in\langle t\rangle+\langle s\rangle+\mathbb{Z}$; hence $|\langle t s\rangle| \leq|\langle t\rangle+\langle s\rangle| \leq|\langle t\rangle|+\mid\langle s\rangle$.) Put $\theta(t)=|\langle t\rangle|$. For every $\beta>0$ let us define $T_{\beta}=\{t \in \mathbb{T}: \theta(t) \leq \beta\}$. Note that $\mathbb{T}_{+}=T_{1 / 4}$ with this notation.
Definition 34. For every Abelian group $G$, every $V \subseteq G$ with $0 \in V$, and every $n \in \mathbb{N}$ we define $\left(1 / 2^{n}\right) V=\left\{x \in V: 2^{k} x \in\right.$ $V \forall k \in\{0,1, \ldots, n\}\}$.

Proposition 35 ([24, Corollary 2$])$. For every $n \in \mathbb{N} \cup\{0\}$ and $\beta \in[0,1 / 3)$, we have $\left(1 / 2^{n}\right)\left(T_{\beta}\right)=T_{\beta / 2^{n}}$.

Lemma 36. Let $G$ be a topological Abelian group, and let $\omega: G \rightarrow \mathbb{T}$ be a quasi-character. Suppose that there exist $U \in \mathcal{N}_{0}(G)$ and $\beta \in(0,1 / 3)$ such that $\omega(U) \subseteq T_{\beta}$. Then $\omega$ is continuous at zero.

Proof. Since $\omega$ is a quasi-character, for every $\rho>0$, there exists $W_{\rho} \in \mathcal{N}_{0}(G)$ with $\omega(u)^{2} \overline{\omega(2 u)} \in T_{\rho}$ for every $u \in W_{\rho}$. Fix any $\varepsilon>0$. Let us find $V \in \mathcal{N}_{0}(G)$ with $\omega(V) \subseteq T_{\varepsilon}$.

Fix any $\beta^{\prime} \in(\beta, 1 / 3)$. Find $N \in \mathbb{N}$ with $\beta^{\prime} / 2^{N} \leq \varepsilon$. Put $V=\left(1 / 2^{N}\right) U \cap\left(1 / 2^{N-1}\right) W_{\left(\beta^{\prime}-\beta\right) /\left(N 2^{N-1}\right)}$.

It is enough to prove that

$$
\forall v \in V \quad \forall n \in\{0,1,2, \ldots, N\}, \quad \omega(v)^{2^{n}} \in T_{\beta^{\prime}}
$$

since by Proposition 35, this will imply $\omega(v) \in T_{\beta^{\prime} / 2^{N}} \subseteq T_{\varepsilon}$. Now, for every $n \in\{0,1,2, \ldots, N\}$

$$
\begin{aligned}
\omega(v)^{2^{n}} & \\
= & \left.\omega(v)^{2^{n}} \frac{\bar{\omega}^{2}{ }^{n-1}}{\omega(2 v)}\right)^{2^{n-1}} \\
& =\left(\omega(v)^{2} \overline{\omega(2 v)}\right)^{2^{n-1}} \omega(2 v)^{2^{n-1}} \\
& =\left(\omega(v)^{2} \overline{\omega(2 v)}\right)^{2^{n-1}} \omega(2 v)^{2^{n-1}} \overline{\omega(2 \cdot 2 v)} 2^{n-2} \omega(2 \cdot 2 v)^{2^{n-2}} \\
& =\left(\omega(v)^{2} \overline{\omega(2 v)}\right)^{2^{n-1}}\left(\omega(2 v)^{2} \overline{\omega(2 \cdot 2 v)}\right)^{2^{n-2}} \omega(2 \cdot 2 v)^{2^{n-2}} \\
& =\cdots \\
& =\left(\prod_{j=0}^{n-1}\left(\omega\left(2^{j} v\right)^{2 \frac{\omega\left(2 \cdot 2^{j} v\right)}{\omega}}\right)^{2^{n-j-1}}\right) \omega\left(2^{n} v\right) .
\end{aligned}
$$

Since $v \in\left(1 / 2^{N}\right) U$, we have that $\omega\left(2^{n} v\right) \in T_{\beta}$. Now, for every $j \in\{0, \ldots, n-1\}$ and every $n \in\{0, \ldots, N\}$, $\omega\left(2^{j} v\right)^{2} \overline{\omega\left(2 \cdot 2^{j} v\right)} \in T_{\left(\beta^{\prime}-\beta\right) /\left(N 2^{N-1}\right)}$, since $2^{j} v \in W_{\left(\beta^{\prime}-\beta\right) /\left(N 2^{N-1}\right)}$. Thus $\left(\omega\left(2^{j} v\right)^{2} \overline{\omega\left(2 \cdot 2^{j} v\right)}\right)^{2^{n-j-1}} \in T_{\left(\beta^{\prime}-\beta\right) / N}$, and we deduce $\prod_{j=0}^{n-1}\left(\omega\left(2^{j} v\right)^{2} \overline{\omega\left(2 \cdot 2^{j} v\right)}\right)^{2^{n-j-1}} \in T_{\beta^{\prime}-\beta}$. This implies that $\omega(v)^{2^{n}} \in T_{\beta^{\prime}}$.

Corollary 37. A quasi-character $\omega: G \rightarrow \mathbb{T}$ is approximable if and only if there exist $U \in \mathcal{N}_{0}(G), \beta \in(0,1 / 3)$ and an algebraic character $\chi \in \operatorname{Hom}(G, \mathbb{T})$ such that $(\omega \bar{\chi})(U) \subseteq T_{\beta}$. Moreover any such $\chi$ approximates $\omega$.

Lemma 38 ([7, Lemma 6]). Let $G$ be an Abelian group (no topology is assumed), and let $\omega: G \rightarrow \mathbb{T}$ be any mapping such that for some $\beta<1 / 3, \omega(x+y) \overline{\omega(x) \omega(y)} \in T_{\beta} \forall x, y \in$ $G$. Then there is a unique character $\chi: G \rightarrow \mathbb{T}$ such that $\omega(x) \overline{\chi(x)} \in T_{\beta} \forall x \in G$. 
Theorem 39. Let $\mu$ be a nonatomic $\sigma$-finite measure on a set $\Delta$. Let $L_{0}:=L_{0}(\mu)$ be the space of all measurable functions on $\Delta$ with the norm

$$
\|f\|_{0}:=\int_{\Delta} \min \{1,|f(t)|\} d \mu(x) .
$$

Then every quasi-character $\omega: L_{0} \rightarrow \mathbb{T}$ is approximable.

Proof. We can assume, without loss of generality, that $\mu$ is a probability (note that $L_{0}(\mu)$ is topologically isomorphic to $L_{0}(\nu)$, where $\nu$ is a probability with the same null sets as $\mu$ ).

Let $\omega: L_{0} \rightarrow \mathbb{T}$ be a quasi-character, fix $\beta<1 / 3$, and choose $\delta_{0}$ such that $\omega(f+g) \overline{\omega(f) \omega(g)} \in T_{\beta}$ for every $f, g$ with $\|f\|_{0},\|g\|_{0} \leq \delta_{0}$.

Let $\Delta=\bigoplus_{i=1}^{r} \Delta_{i}$ be a partition of $\Delta$ into measurable sets, with $\mu\left(\Delta_{i}\right) \leq \delta_{0}$ for all $1 \leq i \leq r$. Then $L_{0}=\prod_{i=1}^{r} L_{0}\left(\Delta_{i}\right)$ as a topological direct product. For all $f \in L_{0}\left(\Delta_{i}\right)$ we have that

$$
\begin{aligned}
\|f\|_{0} & =\int_{\Delta} \min \{1,|f(t)|\} d \mu(x) \leq \int_{\{t \in \Delta: f(t) \neq 0\}} 1 d \mu(x) \\
& =\mu\{t \in \Delta: f(t) \neq 0\} \leq \mu\left(\Delta_{i}\right) \leq \delta_{0} .
\end{aligned}
$$

Call $\omega_{i}$ the restriction of $\omega$ to each $L_{0}\left(\Delta_{i}\right)$. As $\omega_{i}(f+$ $g) \overline{\omega_{i}(f) \omega_{i}(g)} \in T_{\beta}$ for every $1 \leq i \leq r$ and $f, g \in L_{0}\left(\Delta_{i}\right)$, we can apply Lemma 38 to obtain unique characters $\chi_{i}$ : $L_{0}\left(\Delta_{i}\right) \rightarrow \mathbb{T}$ such that $\omega_{i}(f) \overline{\chi_{i}(f)} \in T_{\beta}$ for every $f \in L_{0}\left(\Delta_{i}\right)$. By Lemma 36, we have that each $\omega_{i} \bar{\chi}_{i}$ is continuous at the origin of $L_{0}\left(\Delta_{i}\right)$, and, thus, the character $\chi: L_{0} \rightarrow \mathbb{T}$ given by $\chi(f)=\prod_{i=1}^{r} \chi_{i}\left(f_{i}\right)$ (where $f=\sum_{i=1}^{r} f_{i}, f_{i} \in L_{0}\left(\Delta_{i}\right)$ ) approximates $\omega$ near the origin.

Corollary 40. $L_{0}$ is in $S_{\mathrm{TG}}(\mathbb{T})$.

Proof. This follows from Theorem 39 and Corollary 32, since $L_{0}$ is a metrizable group.

Example 41. Let $L_{0}$ be as in Theorem 39. Fix a discrete, nontrivial $D \leq L_{0}$ (e.g., a copy of $\mathbb{Z}$ ). Note that $L_{0}$ does not have any nontrivial continuous character, and in particular $D$ is not dually embedded in $L_{0}$. Using Theorem 21 we deduce that $L_{0} / D$ is not in $S_{\mathrm{TG}}(\mathbb{T})$. Since $L_{0} \in S_{\mathrm{TG}}(\mathbb{T})$, this example shows that being in $S_{\mathrm{TG}}(\mathbb{T})$ is not preserved by local isomorphisms (compare with Corollary 14).

Let $(G, \tau)$ be a topological group. We say that $(G, \tau)$ is a protodiscrete group (or that the topology $\tau$ is linear) if it has a basis of neighborhoods of 0 formed by open subgroups. Note that protodiscrete Hausdorff groups are exactly the subgroups of products of discrete groups.

Proposition 42. Let $G$ be a protodiscrete topological Abelian group. Every quasi-character of $G$ is approximable.

Proof. Let $\omega: G \rightarrow \mathbb{\mathbb { }}$ be a quasi-character. There exists an open subgroup $U \leq G$ such that $\omega(a+b) \overline{\omega(a) \omega(b)} \in \mathbb{T}_{+}$for every $a, b \in U$. Using Lemma 38 we deduce that there exists an algebraic character $\chi: U \rightarrow \mathbb{T}$ with $\omega(u) \overline{\chi(u)} \in \mathbb{T}_{+}$for every $u \in U$. Now Corollary 37 implies that any algebraic extension of $\chi$ approximates $\omega$.

Corollary 43. Every protodiscrete, metrizable group is in $S_{\mathrm{TG}}(\mathbb{T})$.

Proof. This follows from Proposition 42 and Corollary 32.

Example 44. Countable products of discrete Abelian groups belong to $S_{\mathrm{TG}}(\mathbb{T})$.

\section{Acknowledgments}

The authors acknowledge the financial support of Spanish MICINN (Grant MTM2009-14409-C02-01), and of University of Navarra (PIUNA Grant). The third listed author acknowledges support of Consellería de Educación e Ordenación Universitaria (Xunta de Galicia, Grant CN2011/002) and FEDER funds.

\section{References}

[1] N. J. Kalton, N. T. Peck, and J. W. Roberts, An F-Space Sampler, London Mathematical Society Lecture Note Series, Cambridge University Press, Cambridge, UK, 1984.

[2] M. Bruguera and M. Tkachenko, "The three space problem in topological groups," Topology and Its Applications, vol. 153, no. 13, pp. 2278-2302, 2006.

[3] J. M. F. Castillo, "On the "three-space" problem for locally quasiconvex topological groups," Archiv der Mathematik, vol. 74, no. 4, pp. 253-262, 2000.

[4] N. J. Kalton and N. T. Peck, "Quotients of $L_{p}(0,1)$ for $0 \leq p<1$," Studia Mathematica, vol. 64, no. 1, pp. 65-75, 1979.

[5] F. Cabello, "Pseudo-characters and almost multiplicative functionals," Journal of Mathematical Analysis and Applications, vol. 248, no. 1, pp. 275-289, 2000.

[6] F. Cabello, "Quasi-additive mappings," Journal of Mathematical Analysis and Applications, vol. 290, no. 1, pp. 263-270, 2004.

[7] F. Cabello Sánchez, "Quasi-homomorphisms," Fundamenta Mathematicae, vol. 178, no. 3, pp. 255-270, 2003.

[8] W. Banaszczyk, Additive Subgroups of Topological Vector Spaces, vol. 1466 of Lecture Notes in Mathematics, Springer, Berlin, Germany, 1991.

[9] S. A. Morris, Pontryagin Duality and the Structure of Locally Compact Abelian Groups, Cambridge University Press, Cambridge, UK, 1977, London Mathematical Society Lecture Note Series, No. 29.

[10] M. Bruguera and E. Martín-Peinador, "Open subgroups, compact subgroups and Binz-Butzmann reflexivity," Topology and Its Applications, vol. 72, no. 2, pp. 101-111, 1996.

[11] P. Domański, "On the splitting of twisted sums, and the threespace problem for local convexity," Studia Mathematica, vol. 82, no. 2, pp. 155-189, 1985.

[12] M. Ribe, "Examples for the nonlocally convex three space problem," Proceedings of the American Mathematical Society, vol. 73, no. 3, pp. 351-355, 1979.

[13] J. W. Roberts, "A nonlocally convex F-space with the HahnBanach approximation property," in Banach Spaces of Analytic Functions, vol. 604 of Lecture Notes, pp. 76-81, Springer, Berlin, Germany, 1977. 
[14] S. Warner, Topological Fields, vol. 157 of Mathematics Studies, North-Holland, Amsterdam, The Netherlands, 1989.

[15] E. Hewitt and K. A. Ross, Abstract Harmonic Analysis I, Springer, Berlin, Germany, 2nd edition, 1979.

[16] W. W. Comfort and K. A. Ross, "Topologies induced by groups of characters," Fundamenta Mathematicae, vol. 55, pp. 283-291, 1964.

[17] S. Ardanza-Trevijano and M. J. Chasco, "The Pontryagin duality of sequential limits of topological abelian groups," Journal of Pure and Applied Algebra, vol. 202, no. 1-3, pp. 11-21, 2005.

[18] N. Th. Varopoulos, "Studies in harmonic analysis," Proceedings of the Cambridge Philosophical Society, vol. 60, pp. 465-516, 1964.

[19] J. Galindo and S. Hernández, "The concept of boundedness and the Bohr compactification of a MAP abelian group," Fundamenta Mathematicae, vol. 159, no. 3, pp. 195-218, 1999.

[20] J. B. Reade, "A theorem on cardinal numbers associated with inductive limits of locally compact Abelian groups," Proceedings of the Cambridge Philosophical Society, vol. 61, pp. 69-74, 1965.

[21] S. Hernández, "A theorem on cardinal numbers associated with $\mathscr{L}_{\infty}$ abelian groups," Mathematical Proceedings of the Cambridge Philosophical Society, vol. 134, no. 1, pp. 33-39, 2003.

[22] L. J. Sulley, "On countable inductive limits of locally compact abelian groups," Journal of the London Mathematical Society, vol. 5, no. 2, pp. 629-637, 1972.

[23] R. Venkataraman, "Characterization, structure and analysis on abelian $\mathscr{L}_{\infty}$ groups," Monatshefte für Mathematik, vol. 100, no. 1, pp. 47-66, 1985.

[24] M. J. Chasco and X. Domínguez, "Topologies on the direct sum of topological abelian groups," Topology and Its Applications, vol. 133, no. 3, pp. 209-223, 2003. 


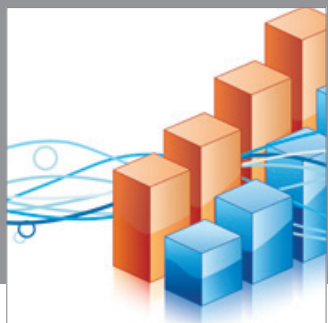

Advances in

Operations Research

mansans

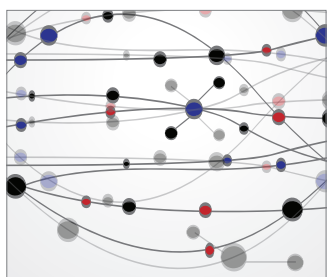

The Scientific World Journal
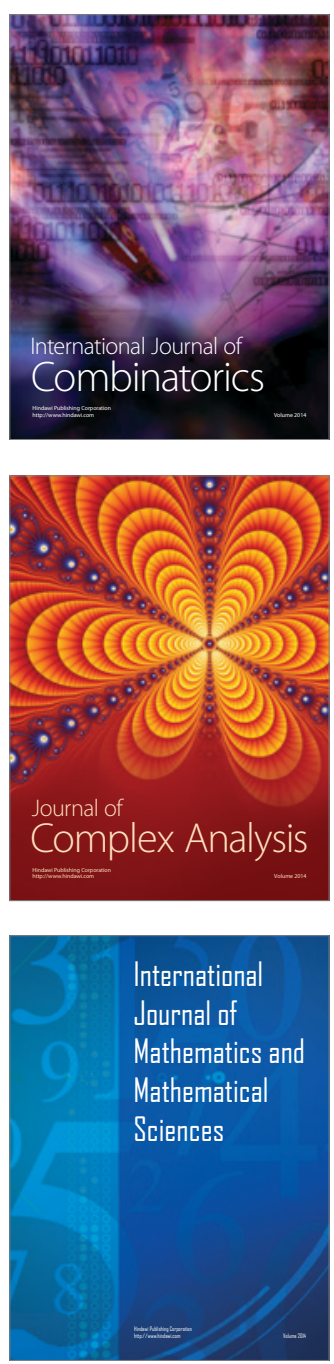
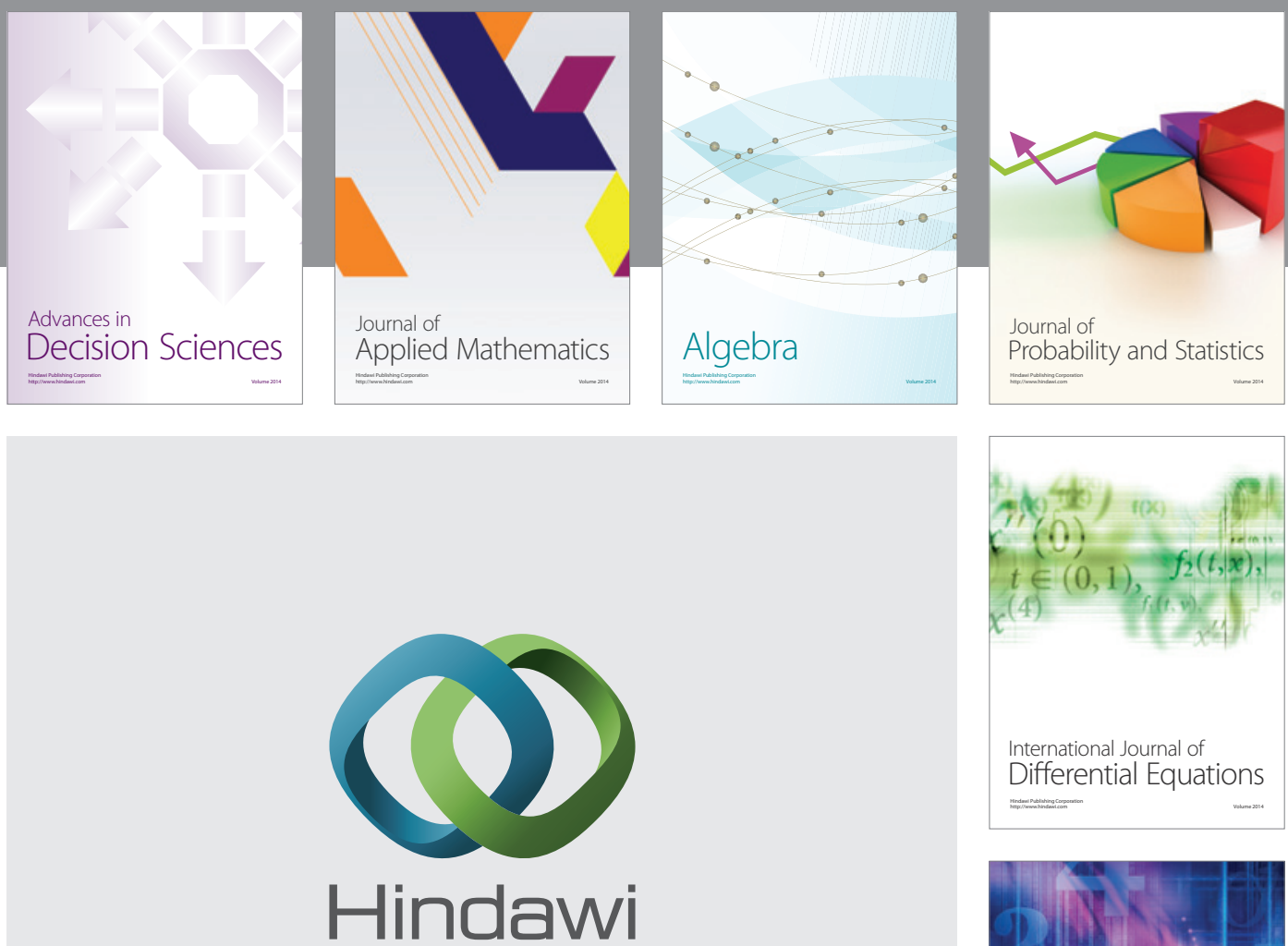

Submit your manuscripts at http://www.hindawi.com
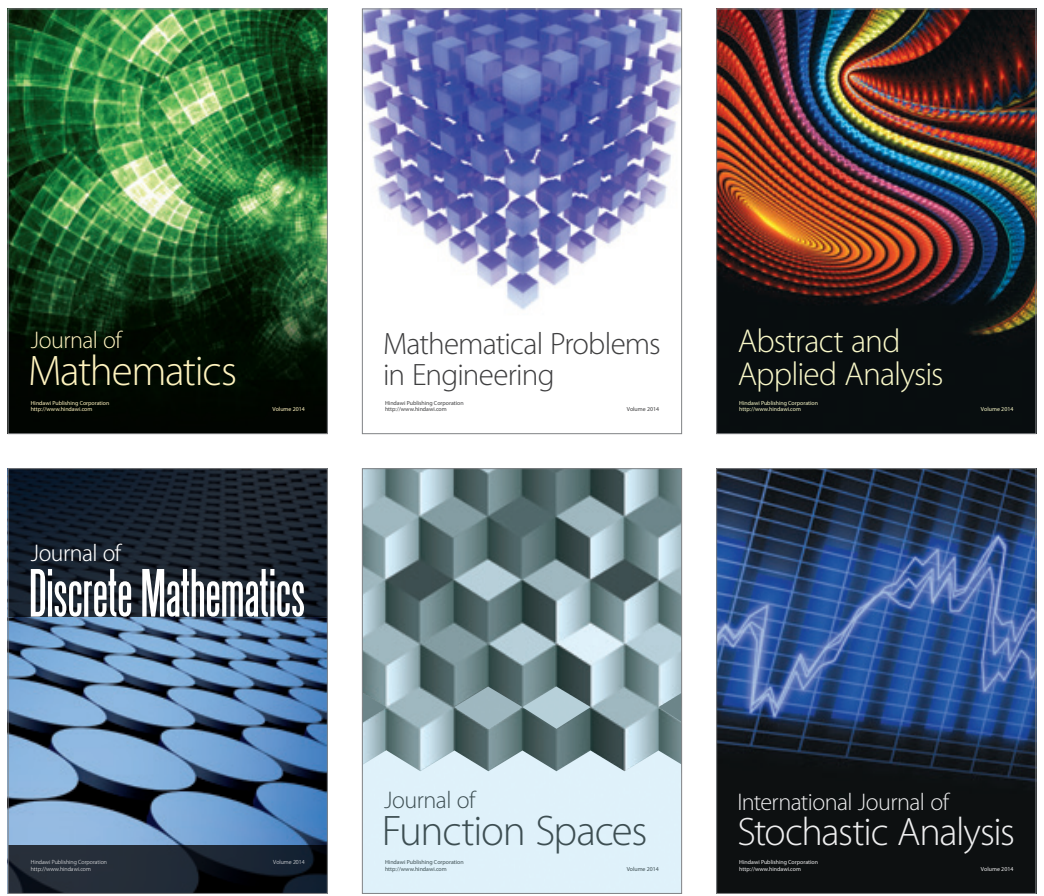

Journal of

Function Spaces

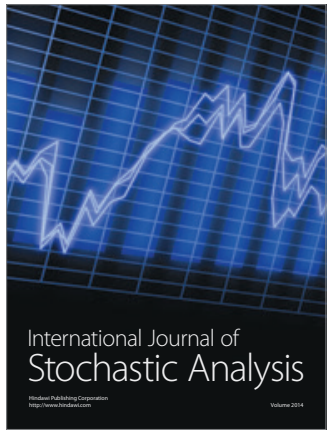

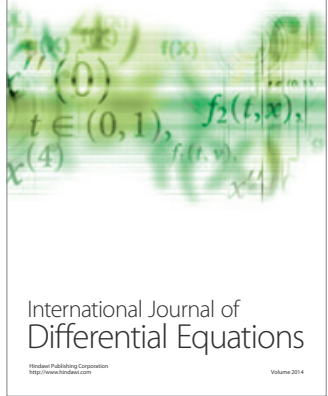
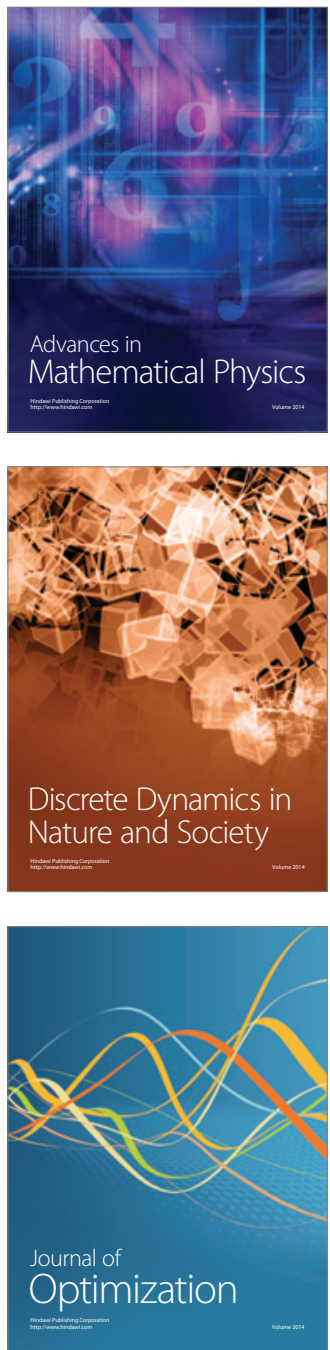